\title{
Imaging and spectroscopy of ten southern galaxies selected from a catalogue of peculiar galaxies
}

\author{
C.J. Donzelli ${ }^{\star, \star \star}$ and D.L. Ferreiro ${ }^{\star}$ \\ IATE, Observatorio Astronómico, Universidad Nacional de Córdoba, Laprida 854, 5000 Córdoba, Argentina
}

Received December 10, 1996; accepted June 16, 1997

\begin{abstract}
We present $B V R I$ broad band photometry and long slit spectroscopy for a sample of ten southern galaxies selected from the Agüero Catalogue of Peculiar Galaxies.

The analysis of the images shows that only four galaxies of the sample are truly peculiar galaxies and four are normal spirals. The remaining two galaxies, a normal spiral and a SO, form an interacting pair. Despite the rather complicated structure of the peculiar galaxies we did not find evidence of mergers in our analysis of images and rotation curves.
\end{abstract}

Almost all galaxies in our sample show star forming regions with very blue colors, $(B-V)$ ranging from 0.2 to 0.6. The SO galaxy shows a very interesting structure in the $V-I$ color map suggesting that star formation events have occurred in concentric annuli centered in the nucleus. In our photometric analysis we did not find characteristics that could distinguish between normal and peculiar galaxies.

We obtained the "pure-emission" line spectra for six galaxies of the sample by subtracting appropriate templates. These templates correspond to ellipticals selected from our library of spectra. This subtraction technique provides a powerful tool to calculate line flux ratios uncontaminated by the underlying stellar population.

Our spectroscopic analysis confirms that the spiral galaxy of the interacting system AM 2054-433 has a LINERtype nucleus. In the normal galaxy ESO 316-29 we find evidence of a LINER nucleus and we report a new Seyfert 2 galaxy, AM 2054-433 N. For this SO galaxy the line flux ratios measured from the pure-emission spectrum are indicative of a Seyfert 2 rather than a LINER nucleus as it was previously reported by other authors.

\footnotetext{
Send offprint requests to: C.J. Donzelli

* Visiting Astronomer at CASLEO Observatory. Complejo Astronómico EL Leoncito is operated under agreement between the Consejo Nacional de Investigaciones Científicas y Técnicas de la Replública Argentina and the National Universities of La Plata, Córdoba and San Juan.

$\star \star$ Visiting Astronomer at the University of Toronto Southern Observatory. Las Campanas, Chile.
}

Three of the four peculiar galaxies in our sample have much higher star-formation rate than normal galaxies, as measured by $\mathrm{H} \alpha+[\mathrm{NII}]$ line emission. The observed $\mathrm{EW}(\mathrm{H} \alpha+[\mathrm{NII}])$ values for these peculiar galaxies are around $60 \AA$, compared to $22 \AA$ for normal galaxies. Whole-aperture spectra for these peculiar galaxies show very similar $\mathrm{EW}(\mathrm{H} \alpha+[\mathrm{NII}])$ values to those observed in their nuclear region suggesting that a global starburst has occurred. Although our sample is small the results presented suggest that peculiar morphology and global starburst events are closely related.

Key words: galaxies: peculiar - galaxies: photometry - galaxies: starburst — galaxies: interactions

\section{Introduction and sample selection}

As it is noted by Kenney \& Koopmann (1996) many papers in the last years point out that the "morphologyenvironment" relationship (Oemler 1992) may partially be due to galaxy-galaxy interactions that play a fundamental role in galaxy evolution (Kennicutt 1990; Moss \& Whittle 1993; Kenney et al. 1995). Schweizer (1992) proposes, on a basis of observational data, that mergers were the primary formation mechanism for elliptical galaxies and spiral bulges. Moreover, this hypothesis has received new observational support since Dressler et al. (1994) studied distant galaxy clusters. These authors noticed the existence of peculiarities in many disk galaxies that could be attributed to interactions or mergers.

On the other hand, peculiar galaxies such as ring galaxies or amorphous galaxies also show evidence of star formation rates higher than normal galaxies (e.g., Appleton \& Struck-Marcell 1987; Gallagher \& Hunter 1987).

Thus, the study of the properties of peculiar galaxies and the search for evidences of mergers could help to understand the role played by the environment on galaxy 
Table 1. Observed galaxies

\begin{tabular}{|c|c|c|c|c|c|c|c|}
\hline Galaxy & R.A. (2000) & Dec. $(2000)$ & Type & $A_{\mathrm{b}}$ & $m_{\mathrm{b}}$ & $c z\left(\mathrm{~km} \mathrm{~s}^{-1}\right)$ & Other names \\
\hline ESO 316-G029 & 10:07:46.0 & $-41: 20: 00$ & PSBT2? & 0.80 & $14.65 \pm 0.14$ & $5668 \pm 62$ & IRAS $10056-4105$ \\
\hline NGC $3250 \mathrm{E}$ & $10: 29: 00.9$ & $-40: 04: 59$ &. $\mathrm{SXS} 6 *$ & 0.48 & $13.19 \pm 0.14$ & $2570 \pm 19$ & $\begin{array}{l}\text { ESO } 317-\text { G } 034 \\
\text { IRAS } 10268-3949\end{array}$ \\
\hline ESO 317-G041 & $10: 31: 22.9$ & $-42: 03: 38$ &. $\mathrm{SBR} 4 * \mathrm{P}$ & 0.56 & $14.42 \pm 0.14$ & $5793 \pm 10$ & IRAS $10292-4148$ \\
\hline NGC 3318 & $10: 37: 15.7$ & $-41: 37: 42$ & .SXT3.. & 0.35 & $12.71 \pm 0.11$ & $2768 \pm 79$ & $\begin{array}{l}\text { ESO } 317-G 052 \\
\text { IRAS } 10350-4122\end{array}$ \\
\hline NGC 3378 & $10: 46: 43.0$ & $-40: 00: 56$ &. S...4*. & 0.40 & $13.48 \pm 0.14$ & $5186 \pm 19$ & $\begin{array}{l}\text { ESO 318- G } 012 \\
\text { IRAS 10444-3945 }\end{array}$ \\
\hline ESO 266-G015 & $11: 40: 56.0$ & $-44: 28: 50$ & .S..4.. & 0.41 & $13.18 \pm 0.14$ & $3160 \pm 40$ & IRAS $11384-4412$ \\
\hline ESO 340-G021 & $20: 22: 28.9$ & $-41: 53: 58$ & $. \mathrm{SXR} 2 .$. & 0.15 & $13.88 \pm 0.14$ & $2591 \pm 35$ & IRAS $20191-4203$ \\
\hline AM $2054-433 \mathrm{~N}$ & $20: 57: 49.4$ & $-43: 21: 05$ & .L..0?. & 0.07 & $14.49 \pm 0.14$ & $9778 \pm 5$ & $\begin{array}{l}\text { ESO 286-G } 017 \\
\text { IRAS 2054-4333 }\end{array}$ \\
\hline AM 2054-433 S & $20: 57: 50.3$ & $-43: 22: 23$ & .SBS4? & 0.07 & $14.30 \pm 0.14$ & $9147 \pm 22$ & ESO 286-G018 \\
\hline IC 5325 & $23: 28: 43.2$ & $-41: 19: 58$ & .SXT4.. & 0.04 & $11.98 \pm 0.12$ & $1498 \pm 37$ & $\begin{array}{l}\text { ESO } 347-\text { G018 } \\
\text { IRAS } 23260-4116\end{array}$ \\
\hline
\end{tabular}

evolution. The main goal of this paper is to describe photometric and spectroscopic properties of a sample of peculiar galaxies in order to shed light on these processes.

Objects were first selected by Agüero (1971) from visual inspection to the Palomar Sky Survey prints. In that work, galaxies were considered peculiar if their subsystems were not concentric or coplanar, or showed some characteristics such as a very fragmented structure or the presence of jets, filaments or amorphous and asymmetric arms. From this Catalogue we have selected those galaxies with no reference available in the Third Reference Catalogue of Bright Galaxies, hereafter RC3, (de Vaucouleurs et al. 1991). We also made use of the Nasa Extragalaxtic Database (NED) to avoid selected galaxies with previous photometric and spectroscopic data. From this sample we have considered a randomly selected sub-sample of ten galaxies for which we have obtained $B V R I$ CCDimages and/or spectra. Table 1 lists selected galaxies together with the information given by the RC3.

In the following, Sect. 2 describes the imaging and spectroscopic observations and reduction techniques. Sect. 3 presents the results of photometry and spectroscopy. In Sect. 4 we present a detailed analysis of the photometric and spectroscopic properties of all galaxies of the sample. Finally, Sect. 5 provides a brief final discussion of our results.

\section{Observations and data reduction}

\subsection{Imaging}

CCD images described in the present paper were obtained with the CASLEO 2.15 m Ritchey-Chrètien telescope, San Juan, Argentina, and the University of Toronto Southern Observatory (UTSO) $0.6 \mathrm{~m}$ reflector at Las Campanas, Chile. In both observatories were used Tektronix CCDs with $1024 \times 102424 \mu \mathrm{m}$ pixels (CASLEO) and $512 \times$ $51248 \mu \mathrm{m}$ pixels (UTSO) with image scales 11.1 and $9.4^{\prime \prime} \mathrm{mm}^{-1}$ respectively.

CASLEO data were obtained during two runs: 1995 march 1-5 and august 22 by CJD and DLF. Images were taken under photometric conditions and with seeing ranging between $2 . .5$ and $3^{\prime \prime}$ (FWHM).

Imaging observations were carried out by CJD during one run at UTSO: 1995 June 22-25. Seeing conditions were very good $\left(1^{\prime \prime} 2-1\right.$." 5$)$ and nights were photometric.

We obtained a set of short $(200-600 \mathrm{~s})$ exposures for each galaxy using $B V R I$ filters. Photometric calibration was achieved using observations of at least 10 standard stars selected from Landolt (1983). Estimates of accuracy in the calibrations are $\pm 0.04 \mathrm{mag}$ in $B, \pm 0.06 \mathrm{mag}$ in $(B-V), \pm 0.05 \mathrm{mag}$ in $(V-R)$ and $\pm 0.05 \mathrm{mag}$ in $(R-I)$.

We have used the usual procedures for reducing images and spectra CCD frames (see Donzelli \& Espíndola 1996 for details). For this purpose we have used the IRAF package (developed by NOAO) and routines of our own. 
Table 2. CCD imaging

\begin{tabular}{lcccccc}
\hline Galaxy & Obs. date & $B$ & $V$ & $R$ & $I$ & Telescope \\
& & exp.(s) & exp.(s) & exp.(s) & exp.(s) & \\
\hline ESO 316-G029 & $01 / 03 / 95$ & $2 \times 400$ & $2 \times 300$ & $2 \times 200$ & $2 \times 200$ & CASLEO 2.15 m \\
NGC 3250E & $02 / 03 / 95$ & $2 \times 400$ & $2 \times 300$ & $2 \times 200$ & - & CASLEO 2.15 m \\
NGC 3318 & $01 / 03 / 95$ & $2 \times 400$ & $2 \times 300$ & $2 \times 200$ & $2 \times 200$ & CASLEO 2.15 m \\
NGC 3378 & $02 / 03 / 95$ & $2 \times 400$ & $2 \times 300$ & $2 \times 200$ & $2 \times 200$ & CASLEO 2.15 m \\
ESO 340-G021 & $25 / 06 / 95$ & $3 \times 600$ & $3 \times 400$ & $3 \times 200$ & $3 \times 200$ & UTSO 0.6 m \\
AM 2054-433 N & $22 / 06 / 95$ & $2 \times 600$ & $2 \times 400$ & $2 \times 200$ & $2 \times 200$ & UTSO 0.6 m \\
AM 2054-433 S & $22 / 06 / 95$ & $2 \times 600$ & $2 \times 400$ & $2 \times 200$ & $2 \times 200$ & UTSO 0.6 m \\
IC 5325 & $26 / 06 / 95$ & $2 \times 600$ & $2 \times 400$ & $2 \times 200$ & $2 \times 200$ & UTSO 0.6 m \\
\hline
\end{tabular}

Table 3. Spectroscopic observations

\begin{tabular}{lccc}
\hline Galaxy & Obs. Date & Exp.(s) & Slit Orientation \\
\hline ESO 316-G029 & $04 / 03 / 95$ & $3 \times 600$ & E-W \\
NGC 3250E & $04 / 03 / 95$ & $3 \times 600$ & major axis \\
ESO 317-G041 & $05 / 03 / 95$ & $4 \times 600$ & major axis \\
NGC 3318 & $04 / 03 / 95$ & $4 \times 600$ & E-W \\
NGC 3378 & $05 / 03 / 95$ & $4 \times 600$ & E-W \\
ESO 266-G015 & $05 / 03 / 95$ & $4 \times 600$ & major axis \\
ESO 340-G021 & $22 / 08 / 95$ & $5 \times 600$ & E-W \\
AM 2054-433 N & $22 / 08 / 95$ & $4 \times 600$ & major axis \\
AM 2054-433 S & $22 / 08 / 95$ & $4 \times 600$ & major axis \\
IC 5325 & $22 / 08 / 95$ & $5 \times 600$ & E-W \\
\hline
\end{tabular}

Table 2 summarizes observation dates, expositions and telescope used.

\subsection{Spectroscopy}

Two-dimensional spectroscopy was carried out with the REOSC Spectrograph at CASLEO $2.15 \mathrm{~m}$ telescope in the runs described above. The spectrograph has attached the same CCD used for images. Spectra in the wavelength range of $3600-7000 \AA$ were taken through a slit aperture of 2.5 long and $33^{\prime \prime} 0$ wide. We use a 300 line $\mathrm{mm}^{-1}$ grating that implies a resolution of $3.3 \AA$. At least three exposures of $600 \mathrm{~s}$ each were obtained for the galaxies with the slit oriented along the major axis or in the $\mathrm{E}-\mathrm{W}$ direction centered in the nucleus.

Spectra were wavelength calibrated using comparison lines from a He-Ne-Ar lamp and calibration accuracy was estimated $<15 \mathrm{~km} \mathrm{~s}^{-1}$.

At least 4 standard stars were observed each night to flux calibrate the spectra. These stars were selected from the Catalogue of Southern Spectrophotometric Standards (Stone \& Baldwin 1983). We estimate an rms of 0.06 magnitudes for residuals of the system sensitivity function fitting. Nevertheless, relative fluxes are probably only good to $\sim 15 \%$ due to inaccuracies in the continuum subtraction and deblending technique. Standard data reduction techniques were used to process the data mainly CCDRED and LONGSLIT packages in the IRAF package.

All spectra are corrected for galactic absorption using $A_{\mathrm{v}}$ values taken from the RC3. Table 3 lists the observation date, exposure, and slit orientation for each galaxy.

\section{Results}

\subsection{Surface photometry}

Total magnitudes and color indices were calculated for 8 galaxies of the sample. These values are listed in Table 4 and are not corrected for galactic absorption. $B$ magnitudes are found in good agreement with RC3. 
Table 4. Total magnitudes

\begin{tabular}{lcccc}
\hline Galaxy & $B$ & $B-V$ & $V-R$ & $R-I$ \\
\hline ESO 316-G029 & 14.45 & 0.80 & 0.91 & 0.64 \\
NGC 3250E & 13.18 & 0.75 & 0.46 & - \\
NGC 3318 & 12.79 & 0.72 & 0.54 & 0.33 \\
NGC 3378 & 13.50 & 0.74 & 0.63 & 0.83 \\
ESO 340-G021 & 13.78 & 1.05 & 0.63 & 0.32 \\
AM 2054-433 N & 14.43 & 0.77 & 0.95 & 0.33 \\
AM 2054-433 S & 14.34 & 0.88 & 1.00 & 0.27 \\
IC 5325 & 11.77 & 0.59 & 0.53 & 0.28 \\
\hline
\end{tabular}

$B$ images of the galaxies are presented throughout Figs. 1a-h were North is at top and East to the left. The bar in the lower left indicates 1 arcmin. A suitable image contrast was chosen to show features in the inner and outer regions of the galaxies.

Surface brightness radial profiles were obtained using the ISOPHOTE package within STSDAS (Jedrezejewski 1987). This routine allows to calculate the galaxy inclination through the major and minor axis ratio of the isophote fitting ellipses. $B$ profiles were decomposed into their bulge and disk components using the method described by Shombert \& Bothun (1987). We used for this purpose the NFIT routine implemented in the FITTING package within STSDAS. Prior to the fitting, smoothing over non-axisymmetric features were taken into account.

Table 5. Photometric parameters

\begin{tabular}{lcccccc}
\hline Galaxy & $\mu_{\mathrm{e}}$ & $r_{\mathrm{e}}$ & $B_{o}$ & $D_{\mathrm{L}}$ & $b / a$ & $B_{\mathrm{D}}$ \\
\hline & & & & & & \\
ESO 316-G029 & 25.1 & 12.0 & 25.7 & 47.9 & 0.4 & 0.35 \\
NGC 3250E & - & - & 22.7 & 15.7 & 0.7 & - \\
NGC 3318 & 21.7 & 2.5 & 23.7 & 33.0 & 0.5 & 0.13 \\
NGC 3378 & - & - & 22.0 & 11.3 & 0.8 & - \\
ESO 340-G021 & 24.7 & 3.0 & 23.1 & 12.7 & 0.8 & 0.05 \\
AM 2054-433 N & 24.5 & 2.0 & 23.5 & 10.5 & 0.5 & 0.05 \\
AM 2054-433 S & 25.8 & 15.0 & 26.0 & 45.0 & 0.2 & 0.48 \\
IC 5325 & 21.9 & 1.5 & 22.6 & 32.3 & 0.7 & 0.02 \\
\hline
\end{tabular}

$B V R I$ and decomposition profiles are shown in Figs. 2a-h were mag $\operatorname{arcsec}^{-2}$ versus radius, in arcsec are plotted. Error bars are only displayed as a reference for the $B$-profile. The resulting structure parameters for both the disk and bulge decomposition, $\mu_{\mathrm{e}}, r_{\mathrm{e}}, B_{0}$ (corrected for the inclination of the disk), and $D_{\mathrm{L}}$, together with the $B / D$
Table 6. Absorption features

\begin{tabular}{lcccc}
\hline Galaxy & CaII-K & CN & CH-G & MgI+MgH \\
& $3393 \AA$ & $4200 \AA$ & $4301 \AA$ & $5175 \AA$ \\
& EW $(\AA)$ & EW $(\AA)$ & EW $(\AA)$ & EW $(\AA)$ \\
\hline ESO 316-G029 & 10.1 & 10.2 & 7.7 & 6.6 \\
Template & 13.1 & 9.0 & 7.5 & 5.2 \\
NGC 3250E & 5.6 & 3.2 & 3.4 & 2.3 \\
Template & 4.5 & 2.4 & 2.2 & 3.2 \\
& & & & \\
ESO 317-G041 & 13.7 & 7.5 & 6.5 & 3.5 \\
Template & 13.1 & 9.0 & 7.5 & 5.2 \\
& & & & \\
ESO 266-G015 & 5.5 & 5.7 & 4.2 & 3.1 \\
Template & 5.5 & 5.5 & 4.9 & 3.7 \\
& & & & \\
AM2054-433N & 8.8 & 4.8 & 6.4 & 5.6 \\
Template & 9.9 & 4.6 & 5.9 & 6.2 \\
& & & & \\
AM2054-433S & 9.1 & 10.8 & 8.6 & 6.8 \\
Template & 10.3 & 9.5 & 8.0 & 7.9 \\
\hline
\end{tabular}

light ratio and $b / a$ are listed in Table 5 for those galaxies in which the decomposition technique was possible.

In order to study stellar populations and dust distribution in the galaxies, we also constructed $B-V, B-I$ and $V-R$ color maps. Images were first aligned using the algorithm described by Marston (1988), normalised to the same integration time and rebinned by a $3 \times 3$ box to improve the signal-to-noise ratio. These color maps are discussed in Sect. 4.

We also calculate $(B-V)$ colors of various knots present in most galaxies of the sample. These magnitudes were calculated subtracting an appropriate background that takes into account the galaxy luminosity profile. These results are discussed throughout Sect. 4.

\subsection{Spectrophotometry}

For each galaxy all the flux along the slit was combined to create a "whole-aperture" or "integrated" spectrum, and a small aperture of $2 \mathrm{kpc}$ in linear size was also extracted to obtain the spectra of the nuclear region (we use throughout this paper $H_{0}=75 \mathrm{~km} \mathrm{~s}^{-1} \mathrm{Mpc}^{-1}$ ). Whole-aperture spectra were mainly used to determine the star formation activity in the outer regions of the galaxies and to classify them according to the criteria given by Kennicutt (1992).

From the nuclear spectra we were able to obtain "pureemission" spectra using a similar method described by Bica (1988). First, we measured the EW of the following absorption features: CaII-K $3393 \AA$, CN $4200 \AA$, CH-G 

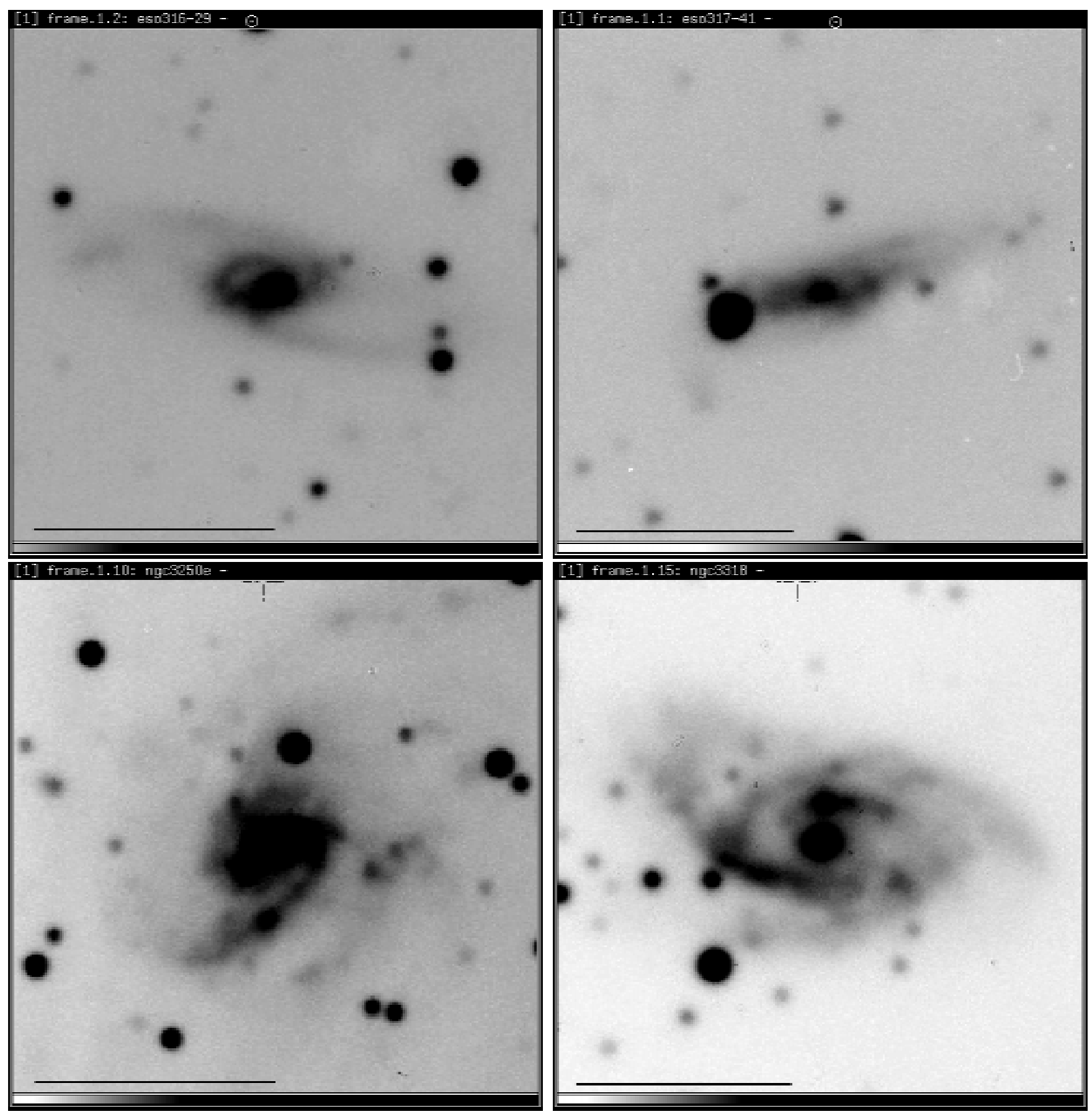

Fig. 1. $B$ images of the sample galaxies. North is at top and East to the left. The bar in the lower left indicates 1 arcmin. a) ESO 316G29, b) NGC3250E, c) ESO 317-G41, d) NGC 3318, e) NGC 3378, f) ESO 340-G21, g) AM 2054-433, h) IC 5325

$4301 \AA$ and $\mathrm{MgI}+\mathrm{MgH} 5175 \AA$. These measurements allowed us to select an appropriated stellar absorption spectrum (template) that reproduces the underlying stellar population. The pure-emission spectrum for each galaxy was then obtained by subtracting the template from the original spectrum. These templates were selected from a database that contains early type galaxy spectra (Donzelli \& Pastoriza 1997). Prior to the subtraction both galaxy and template spectra were normalised to the continuum at $5870 \AA$ A. Reddening of the galaxies was initially calculated choosing appropriate $E(B-V)$ values in order to get a good matching between the galaxy and template continua over the blue region. However, in each case $E(B-V)$ was recalculated using the pure-emission spectra assuming an intrinsic $\mathrm{H} \alpha / \mathrm{H} \beta$ ratio of 2.85 (decrement for case $\mathrm{B}$ Balmer recombination in an optically thin plasma at 

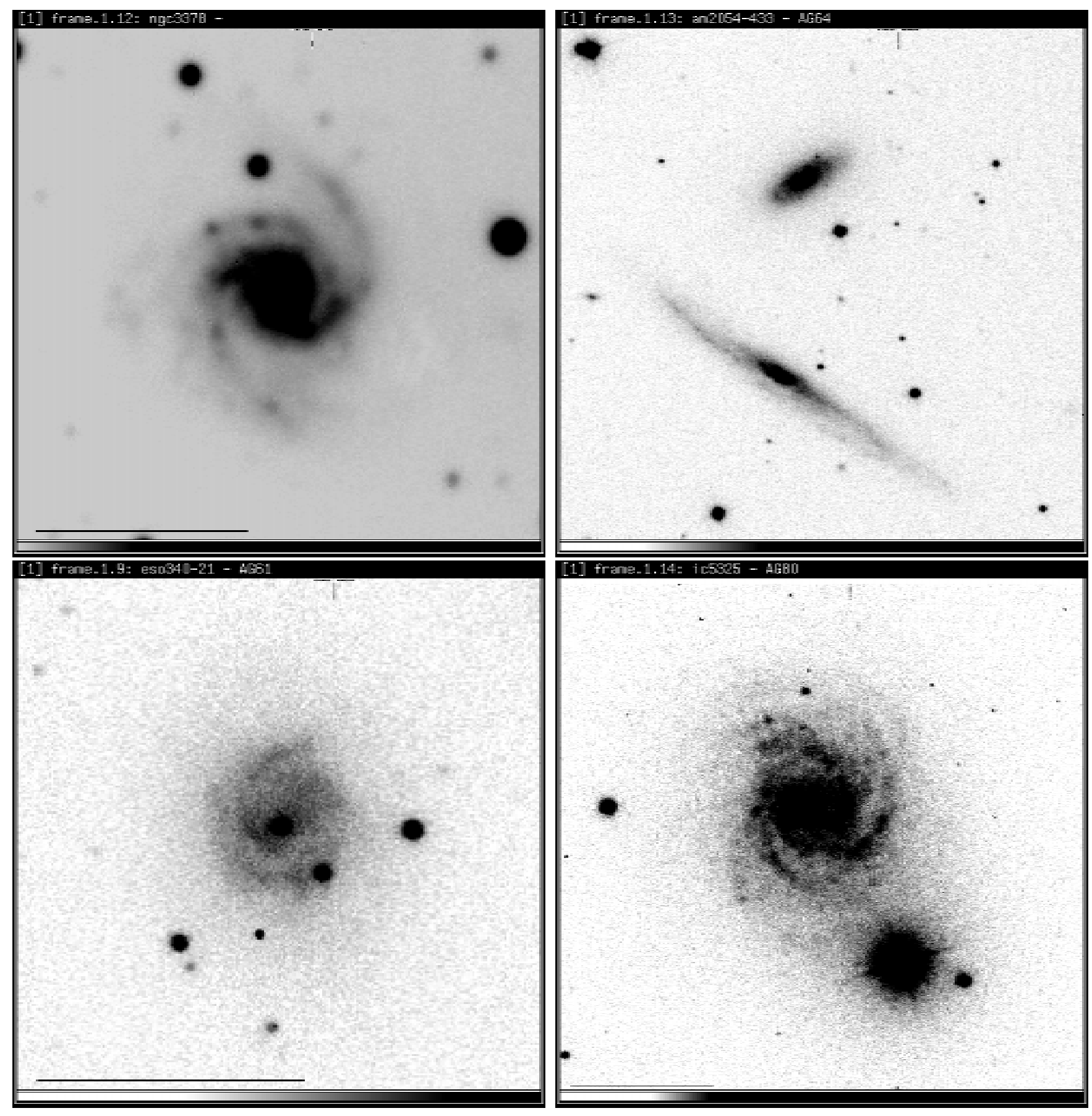

Fig. 1. continued

$T=10^{4} \mathrm{~K}$, Osterbrock 1989). For 6 galaxies of the sample: ESO 316-29, NGC 3250E, ESO 317-41 and the interacting pair AM 2054-433 it was possible to apply this technique. For the remaining galaxies we could not find an appropriate template from the database.

The measured equivalent width of the absorption features for both observed galaxies and templates are listed in Table 6.
In order to classify the spectra using the diagnostic diagrams (Veilleux \& Osterbrock 1987) we use line fluxes measurements for [OII] $3727 \AA, \mathrm{H} \beta$, [OIII] 4959, $5007 \AA$, [OI] $6300 \AA$, [NII] 6548, $6583 \AA$, $\mathrm{H} \alpha$ and [SII] $6717,6731 \AA$, in the pure-emission spectra. Fluxes are extinction corrected using the reddening curve provided by McCall et al. (1985). Table 7 lists the observed $\mathrm{EW}(\mathrm{H} \alpha+$ $[\mathrm{NII}])$ and $\mathrm{EW}([\mathrm{OII}])$ values for both whole-aperture and nuclear spectra. Table 8 gives $E(B-V)$ observed values 

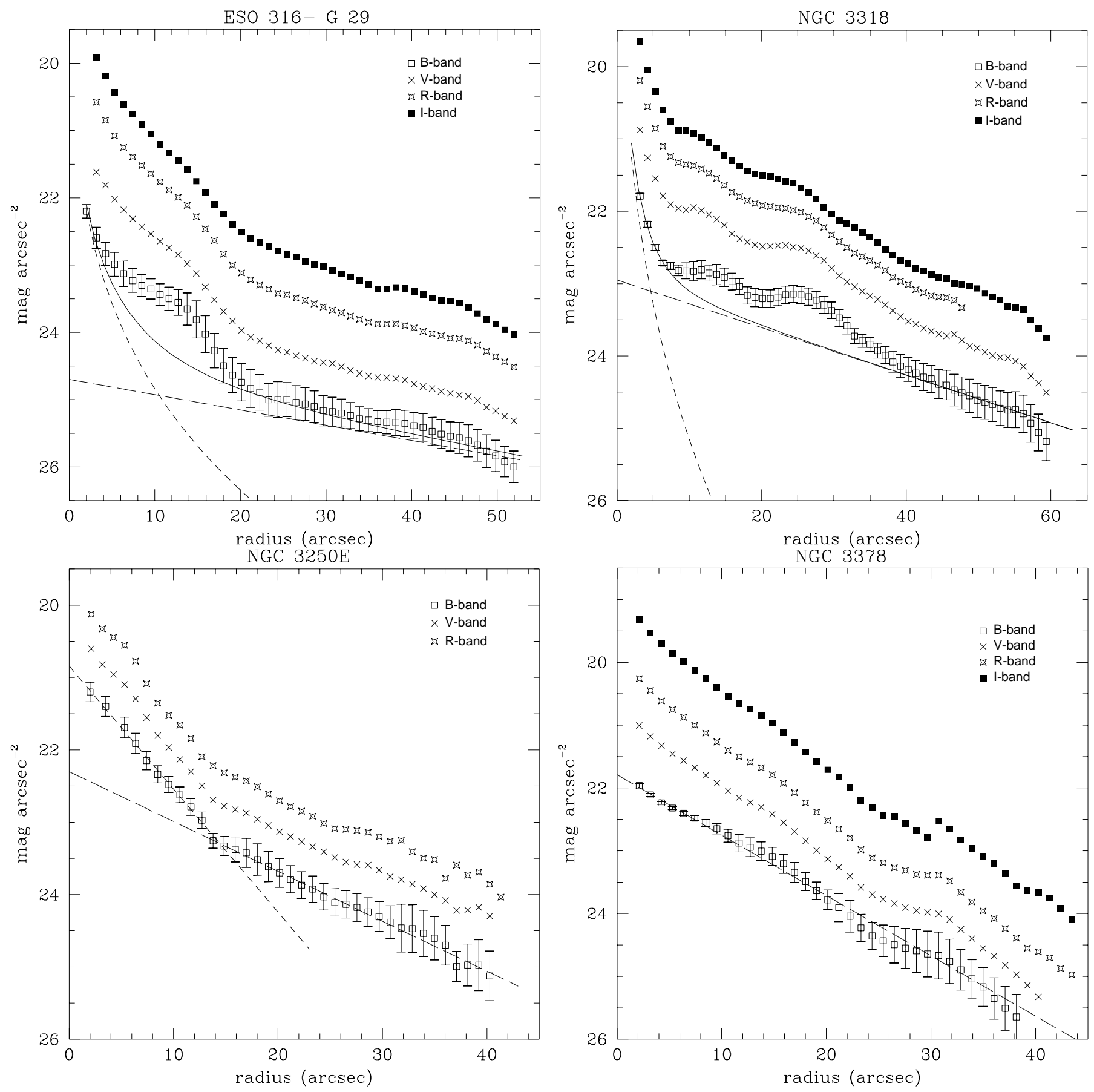

Fig. 2. Luminosity profiles of the sample galaxies. Error bars are only displayed for the $B$-profiles as a reference. The solid line is the best fit to the data for the the sum of a bulge profile (short dashed) plus an exponential disk (long dashed). a) ESO 316-G29, b) NGC 3250E, c) NGC 3318, e) ESO 340-G21, f) AM 2054-433N, g) AM 2054-433S, h) IC 5325

together with the line flux ratios calculated from the pure emission spectra.

Radial velocities were also measured from the nuclear spectra using the cross-correlation technique developed by Tonry \& Davies (1979) as implemented in the RV program in the IRAF package. Typical rms values obtained were around $60 \mathrm{~km} \mathrm{~s}^{-1}$. The calculated radial velocities are also listed in Table 7.

We also determine the rotation curve of the galaxies that show extended emission. For this purpose we use $\mathrm{H} \alpha$ and [NII] $6583 \AA$ emission lines and the cross-correlation technique. Rotation curves are shown in Figs. 3a-e, where is plotted the velocity (in $\mathrm{km} \mathrm{s}^{-1}$ ), corrected for galaxy 

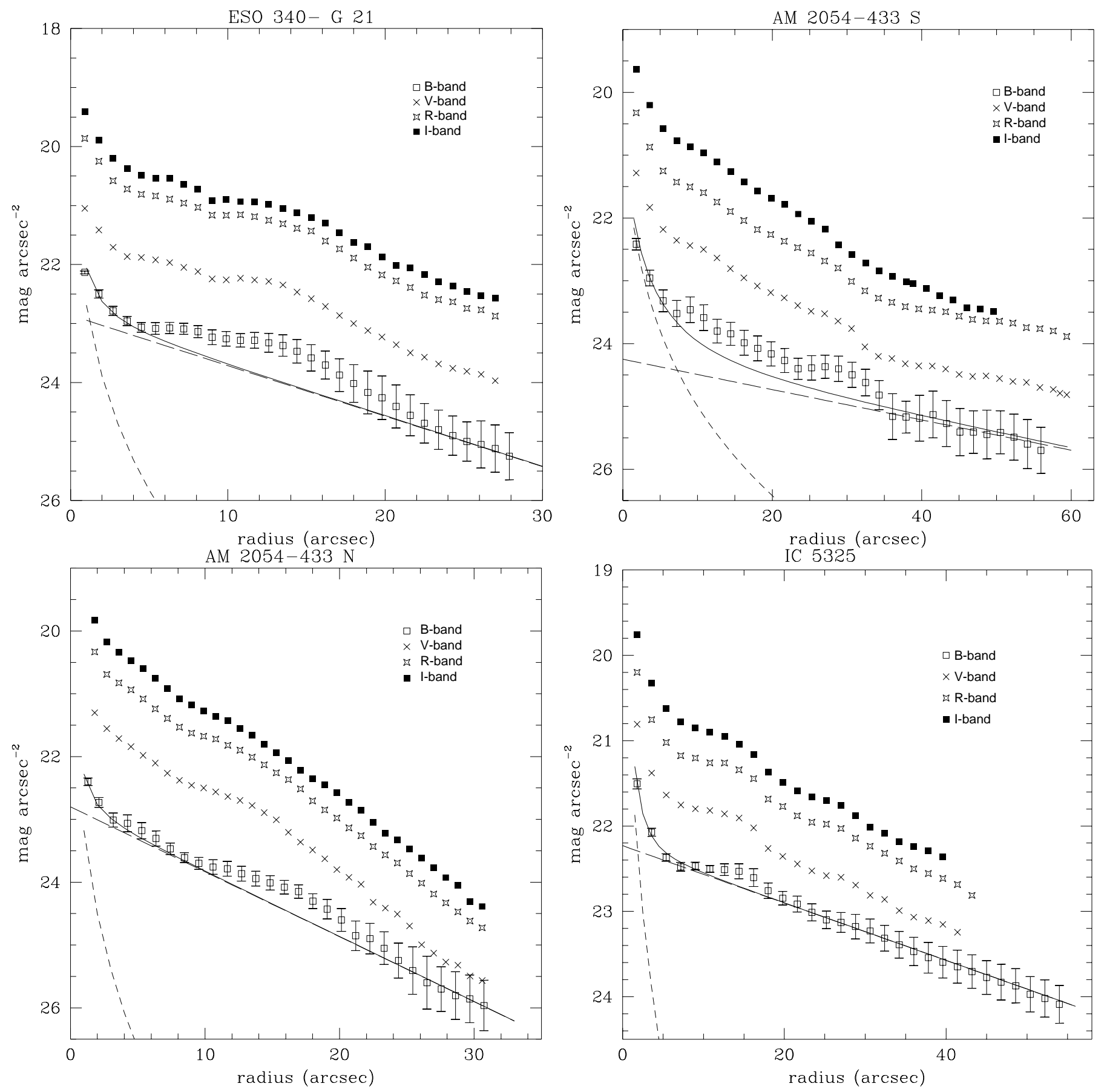

Fig. 2. continued

inclination, versus radius (in $\mathrm{kpc}$ ) where kinematical centers are adopted as origin of coordinates. A comparison of our results for the spiral galaxy AM 2054-433 with those published by Persic \& Salucci 1995 are found in a very good agreement.

Upper and lower panels of Figs. 4a-j plot the wholeaperture and the nuclear spectra respectively. Wavelength is in angstroms and flux in units of $10^{-15} \mathrm{ergs} \mathrm{cm}^{-2} \mathrm{~s}^{-1}$ $\AA^{-1}$ and it is also indicated in the whole-aperture spectra the linear size, in kpc, of the integrated region. All spectra are corrected by redshift and galactic absorption and plotted on a common wavelength scale of $3600-7000 \AA$. We have not corrected the spectra for reddening within the galaxies themselves.

We have also dated the star-formation events for the peculiar galaxies comparing the equivalent width of the Balmer lines with the values taken from a grid of evolutionary synthesis models (Leitherer \& Heckman 1995, 

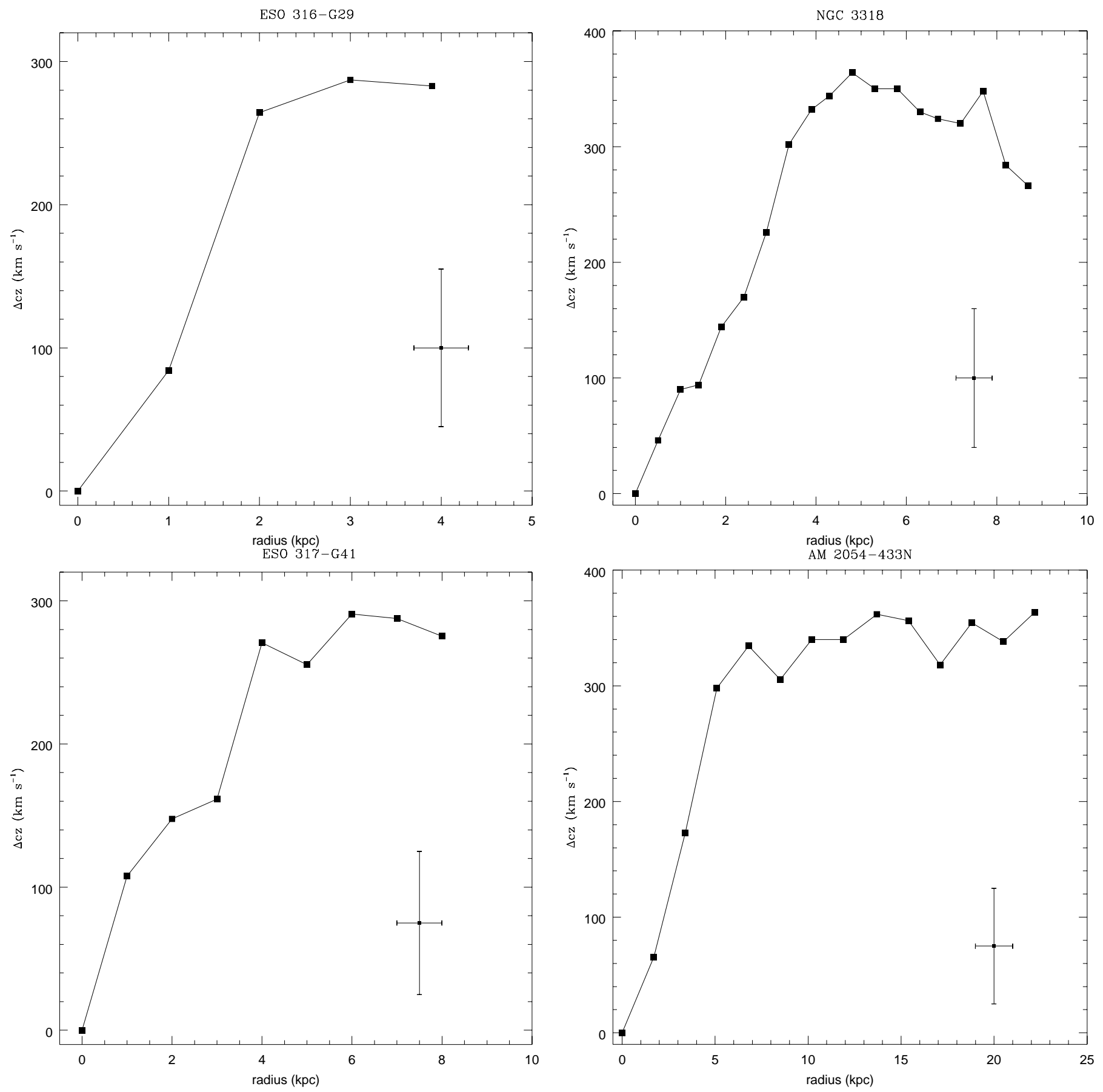

Fig. 3. Rotation curve of the sample galaxies corrected for the galaxy inclination. Kinematical centers were used as origing of coordinates. The cross indicates the velocity and radius uncertainties. a) ESO 316-G29, b) ESO 317-G41, c) NGC 3318, d) AM 2054-433N, e) AM 2054-433S

hereafter $\mathrm{L} \& \mathrm{H})$. These models are based on a population of massive stars $\left(M \geq 30 M_{\odot}\right)$ and consider the typical conditions found in HII regions, HII galaxies, blue compact dwarf galaxies, nuclear starburst and infrared luminous starburst galaxies. It was used as input parameter the $\mathrm{EW}(\mathrm{H} \alpha)$ and we assumed an instantaneous burst model with $\alpha=2.35, M_{\text {up }}=30 M_{\odot}$ and $Z=Z_{\odot}$. We have adopted this model because the $[\mathrm{OIII}] / \mathrm{H} \beta$ observed values are representative of HII regions younger than $15 \mathrm{Myr}$ according to the models of Copetti et al. (1986). The results obtained are analyzed throughout Sect. 4 . 


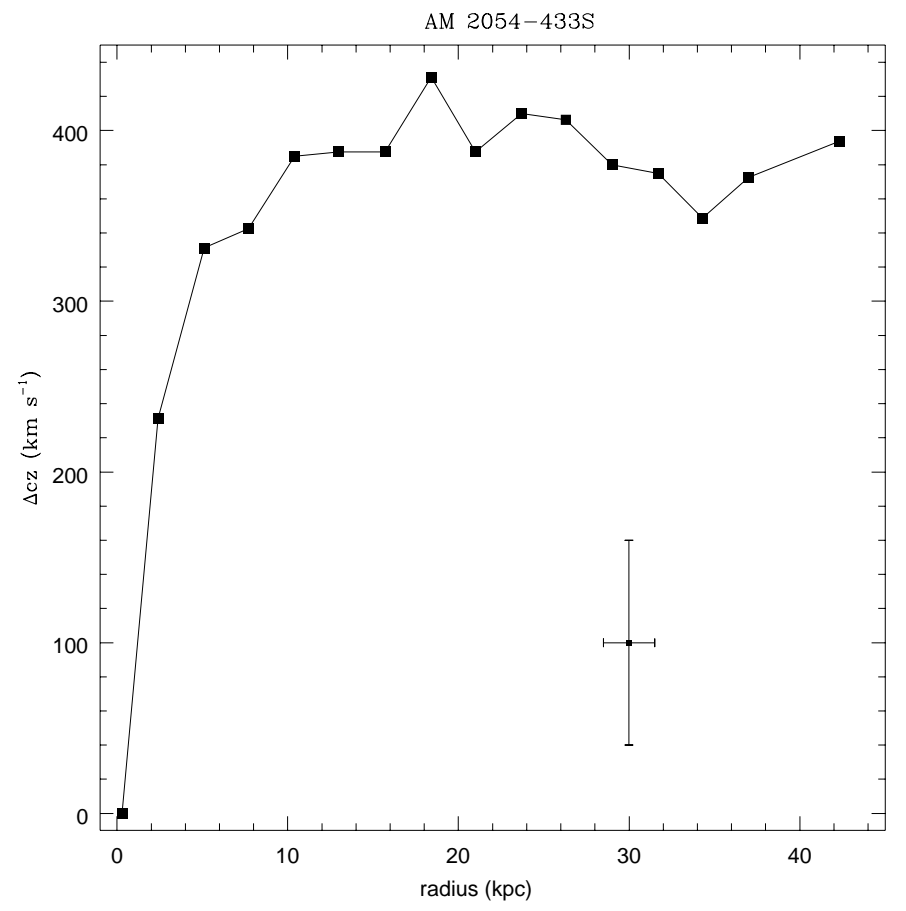

Fig. 3. continued

\section{The galaxies}

ESO 316-G029: Despite the asymmetric ring-like structure in the nuclear region of this galaxy (see Fig. 1a) we classified it as a normal galaxy. It is a barred spiral with two well developed arms. The arms are relatively faint and it is noted that there is a knot at the end of the NE arm. The color of this clump, $(B-V) \sim 0.6$, is indicative that it was probably caused by the disruption of the arm itself.

The luminosity profiles shown in Fig. 2a, exhibit a very uniform behaviour. The feature at $r=15^{\prime \prime}$ is probably due to the presence of the inner ring which is more conspicuous in the $B$ band.

We measured the rotation curve of this galaxy up to a radius of $4 \mathrm{kpc}$. It is seen in Fig. $3 \mathrm{a}$ that the semiamplitude, corrected for the galaxy inclination $(b / a=$ $0.4)$, is $285 \pm 50 \mathrm{~km}^{-1}$.

This galaxy has a LINER-type nucleus as it can be seeing from its spectrum shown in Fig. 4a. Although the observed $[\mathrm{NII}] / \mathrm{H} \alpha$ ratio barely satisfies the definition of a LINER galaxy (Sekinguchi \& Wolstencroft 1992, hereafter S\&W), the spectrum shows relatively strong [OIII] 4959, $5007 \AA$ emission lines. Moreover, the $\mathrm{EW}(\mathrm{H} \alpha+$ $[\mathrm{NII}])=22 \AA$ measured from the nuclear spectrum is high when compared to a typical $\mathrm{Sa}$ - Sab galaxy spectra (Kennicutt 1992). The calculated line flux ratios from the pure-emission spectrum of this galaxy are listed in Table 8. From these data it was derived an internal reddening $E(B-V)=1.25$, the higher value in the whole sample.
NGC 3250 E: Although the structure of this galaxy is rather peculiar (see Fig. 1b), we find no evidences of a merger. The $B$ image shows a very irregular and bright main body wrapped up by three spiral arms. The nucleus is not in the galaxy center and it can be also noticed a conspicuous bar.

The $B-V$ color map of this galaxy shows several very blue knots distributed along the arms. These regions have $(B-V)$ values ranging from 0.1 to 0.4 indicating ongoing star formation. Particularly interesting is the compact region located at the end of the southern arm for which we obtain $(B-V)=0.2$.

As it can be seen in Fig. 2b the luminosity profiles of this galaxy show an interesting property. Two exponential behaviours for $r<15^{\prime \prime}$ and for $r>15^{\prime \prime}$, suggests that the galaxy has two different discs with scale lengths $D_{\mathrm{L}}=$ $2.8 \mathrm{kpc}$ and $D_{\mathrm{L}}=11.1 \mathrm{kpc}$ respectively.

According to the criteria given by Kennicutt (1992), the whole-aperture spectrum of this galaxy corresponds to a starburst galaxy as it can be seen in Fig. 4b. From the nuclear spectrum we obtain $\mathrm{EW}(\mathrm{H} \alpha+[\mathrm{NII}])=60 \AA$, which is about 3 times higher than the corresponding average for Sb/Sc galaxies (Liu \& Kennicutt 1995, hereafter $\mathrm{L} \& \mathrm{~K})$. On the other hand, the whole-aperture and nuclear spectra give similar $\mathrm{EW}(\mathrm{H} \alpha+[\mathrm{NII}])$ values indicating that the galaxy is probably under a global starburst (see Table 7). According to the models of L\&H the age of the burst is around $10 \mathrm{Myr}$.

ESO 317-G041: This is clearly another peculiar galaxy whose elongated main body shows several filaments or tails which are not aligned with major axis (see Fig. 1c).

Due to bad tracking (see the bright star located at the $E$ in Fig. 1c) and calibration problems we could not obtain the luminosity profiles of this galaxy.

The integrated spectrum of this object corresponds to a starburst galaxy (see Fig. 4c). However, the absorption spectrum is more conspicuous than the spectrum of the ESO 317-G041. This fact is particularly evident in the blue, where the Balmer spectrum of early-type young stars have become the dominant spectral feature.

From the nuclear spectrum we obtain $\mathrm{EW}(\mathrm{H} \alpha+$ $[\mathrm{NII}])=58 \AA$, while for the integrated spectrum $\mathrm{EW}(\mathrm{H} \alpha+$ $[\mathrm{NII}])=47 \AA$. These values date the age of the starburst event about $10 \mathrm{Myr}$.

We measured the rotation curve of this galaxy up to a radius of $7 \mathrm{kpc}$. The maximum rotational velocity is $180 \pm$ $50 \mathrm{~km} \mathrm{~s}^{-1}$ and it is reached at $r=4 \mathrm{kpc}$ (see Fig. $3 \mathrm{~b}$ ).

NGC 3318: This is a very peculiar galaxy. The blue image shows a very bright stellar-nucleus located in the middle of a bar that lies along the $\mathrm{N}-\mathrm{S}$ direction (see Fig. 1d). From this bar two well developed and asymmetrical arms emerge. They are very bright, specially the southern one, and they show very blue colors, $(B-V) \sim$ $0.3-0.4$, indicating ongoing star formation. Another interesting feature of this galaxy is a ring-like structure that 

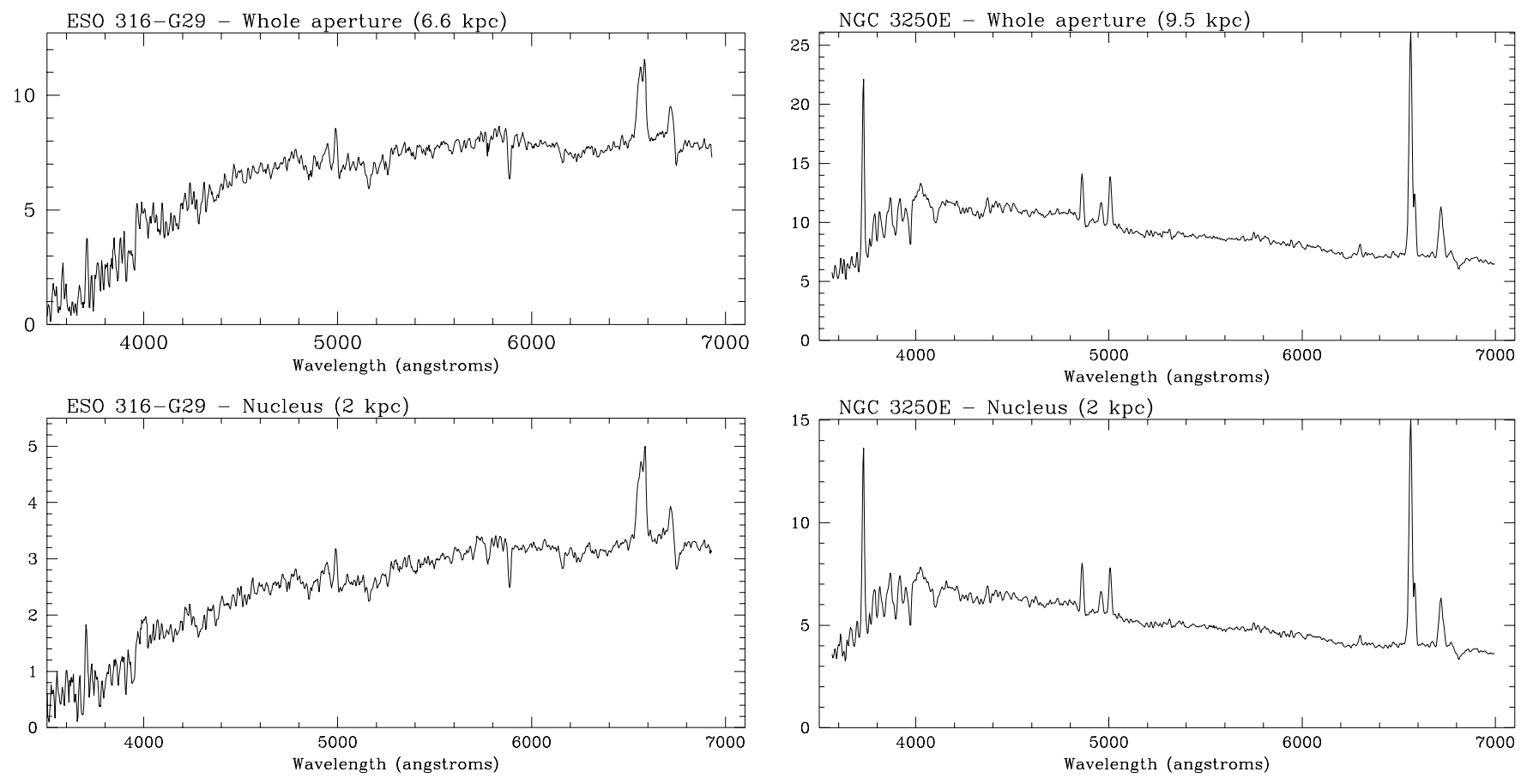

Fig. 4. Whole-aperture (top panel) and nuclear (bottom panel) spectra of the sample galaxies. Flux is in units of $10^{-15} \mathrm{ergs} \mathrm{cm}^{-2} \mathrm{~s}^{-1}$ and wavelenght is in angstronms. a) ESO 316-G29, b) NGC 3250E, c) ESO 317-G41, d) NGC 3318, e) NGC 3378, f) ESO 266-G15, g) ESO 340-G21, h) AM 2054-433N, i) AM2054-433S, j) IC 5325

emerges from the extreme of the southern arm and seems to be in a different plane than the disk.

The luminosity profiles shown in Fig. 2c exhibit many features that are signatures of the rather complicated structure of this galaxy. From the $B-V$ map it can be noticed several knots distributed throughout the galaxy with rather blue colors, $(B-V) \sim 0.4-0.6$.

Despite the peculiar morphology of this galaxy the integrated spectrum corresponds to a $\mathrm{Sb}$ galaxy (see Fig. 4d). We measure $\mathrm{EW}(\mathrm{H} \alpha+[\mathrm{NII}])=26 \AA$, which is typical for this type of galaxies. However, the nuclear and integrated spectra of this galaxy are slightly different. The integrated spectrum shows a stronger [OII] $3727 \AA$ emission line and lower $[\mathrm{NII}] / \mathrm{H} \alpha$ ratio than the nuclear spectrum due to the presence of HII regions outside the nucleus.

Figure $3 \mathrm{c}$ shows the rotation curve up to a radius of $7 \mathrm{kpc}$ where a high value of the maximum velocity, $v_{\max }=$ $340 \pm 50 \mathrm{~km} \mathrm{~s}^{-1}$ is reached at $r=4 \mathrm{kpc}$.

NGC 3378: By inspection to Fig.1e we classify this galaxy as a normal spiral. It shows a faint stellar-nucleus and a bright disk from which three spiral arms emerge. In the $B-V$ map we observe several blue knots distributed throughout the arms. The $(B-V)$ values range from 0.3 to 0.4 indicating ongoing star formation.

The luminosity profiles shown in Fig. 2d exhibit an exponential behaviour in the whole galaxy. The presence of a feature at $r=30^{\prime \prime}$ is due to the contribution of the spiral arms. From the decomposition profiles we measured for this galaxy $D_{\mathrm{L}}=3.5 \mathrm{Kpc}$.

The whole-aperture spectrum corresponds to a $\mathrm{Sa}$ galaxy. This spectrum is mainly dominated by evolved giant stars. However the appearance of emission lines such as [NII] $6548,6583 \AA$ and $\mathrm{H} \alpha$, with equivalent widths of only a few angstroms, indicates the presence of a young stellar population. For the integrated spectra of this galaxy we measure $\mathrm{EW}(\mathrm{H} \alpha+[\mathrm{NII}])=8 \AA$.

ESO 266-G015: Although we could not take images of this galaxy, a preliminary examination can be made from the original prints of Agüero (1971). This is a peculiar galaxy with an irregular and very weak disk from which two filaments of different shape and size emerge. These filaments reassemble spiral arms, the southern being very wide and short, the northern thin and long. The nucleus is not located in the galaxy center.

The whole-aperture spectrum corresponds to a starburst galaxy showing strong $\mathrm{H} \alpha$ as well as [NII] 6548, $6583 \AA$ [OII] $3727 \AA, \mathrm{H} \beta,[\mathrm{OI}] 6300 \AA$, and [SII] 6717, $6731 \AA$ emission lines. The similar observed values of $\mathrm{EW}(\mathrm{H} \alpha+[\mathrm{NII}])$ in both nuclear and integrated spectra indicates that this galaxy is under a global starburst (see Table 7).

ESO 340-G021: From a visual inspection of the $B$ image shown in Fig. If we may conclude that this is a normal Sb galaxy. It shows a stellar-nucleus and a very bright disk when compared to the arms. The arms are 

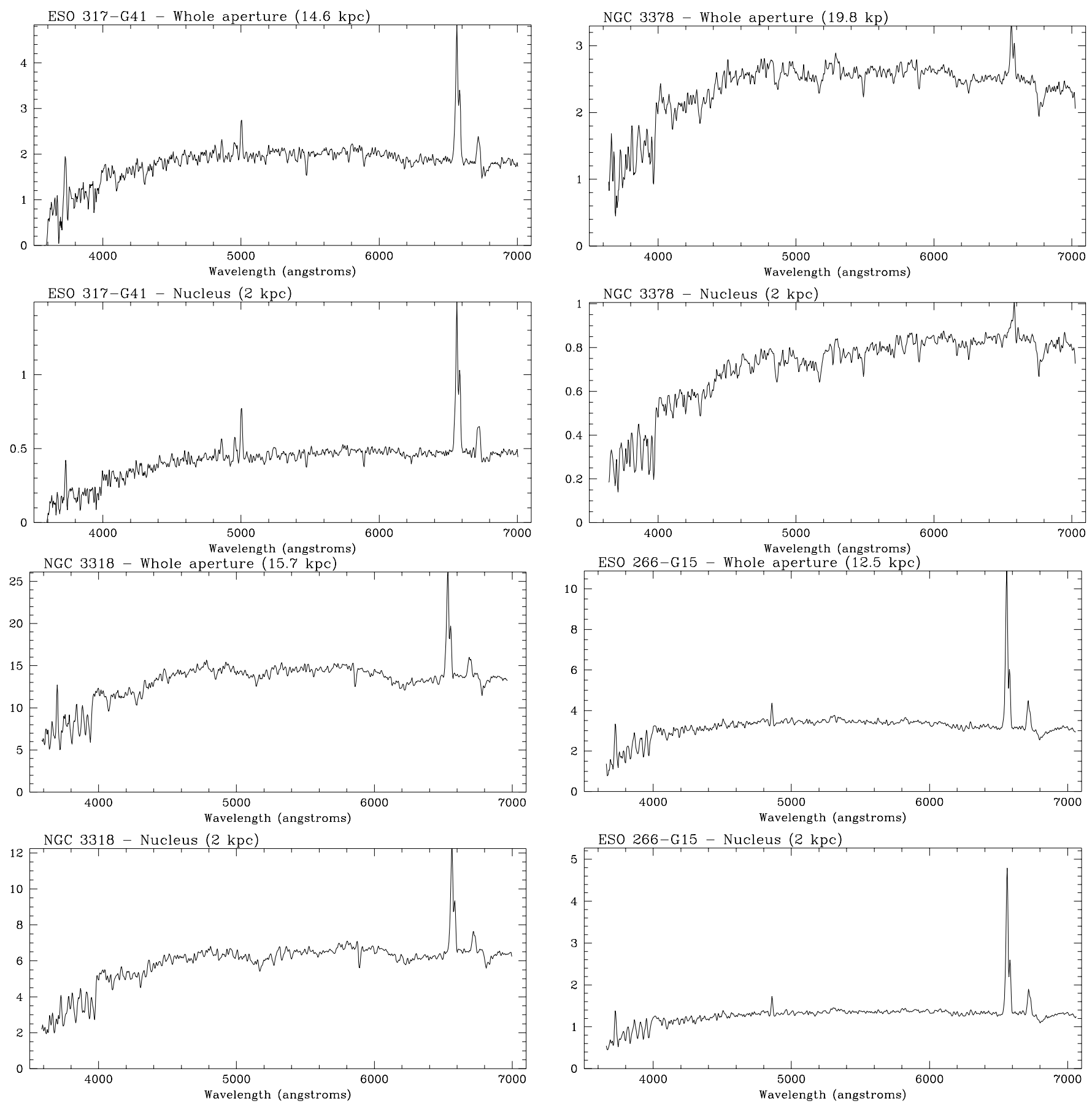

Fig. 4. continued

somewhat weak in the blue and more prominent in the red.

The luminosity profiles shown in Fig. 2e, exhibit the same trend in all bands indicating an homogeneous color throughout the galaxy. The profiles are clearly disk dominated and the observed excess in the exponential law for $5^{\prime \prime}<r<22^{\prime \prime}$ is due to the contribution of the spiral arms.

Both nuclear and integrated spectra show the same signatures and, as in the case of NGC 3318, they corre-

spond to a Sb galaxy (see Fig. 4g). It can also be noted in the spectra a blue continuum from young stars and the G- and K-giant absorption features.

AM 2054-433N: In Fig. 1g can be appreciated that this is a normal SO galaxy, and with AM $2054-433 \mathrm{~S}$ is the only interacting system of the sample. From our images it is distinguished a faint stellar-nucleus from which emerges a small filament towards the NW. The disk is 

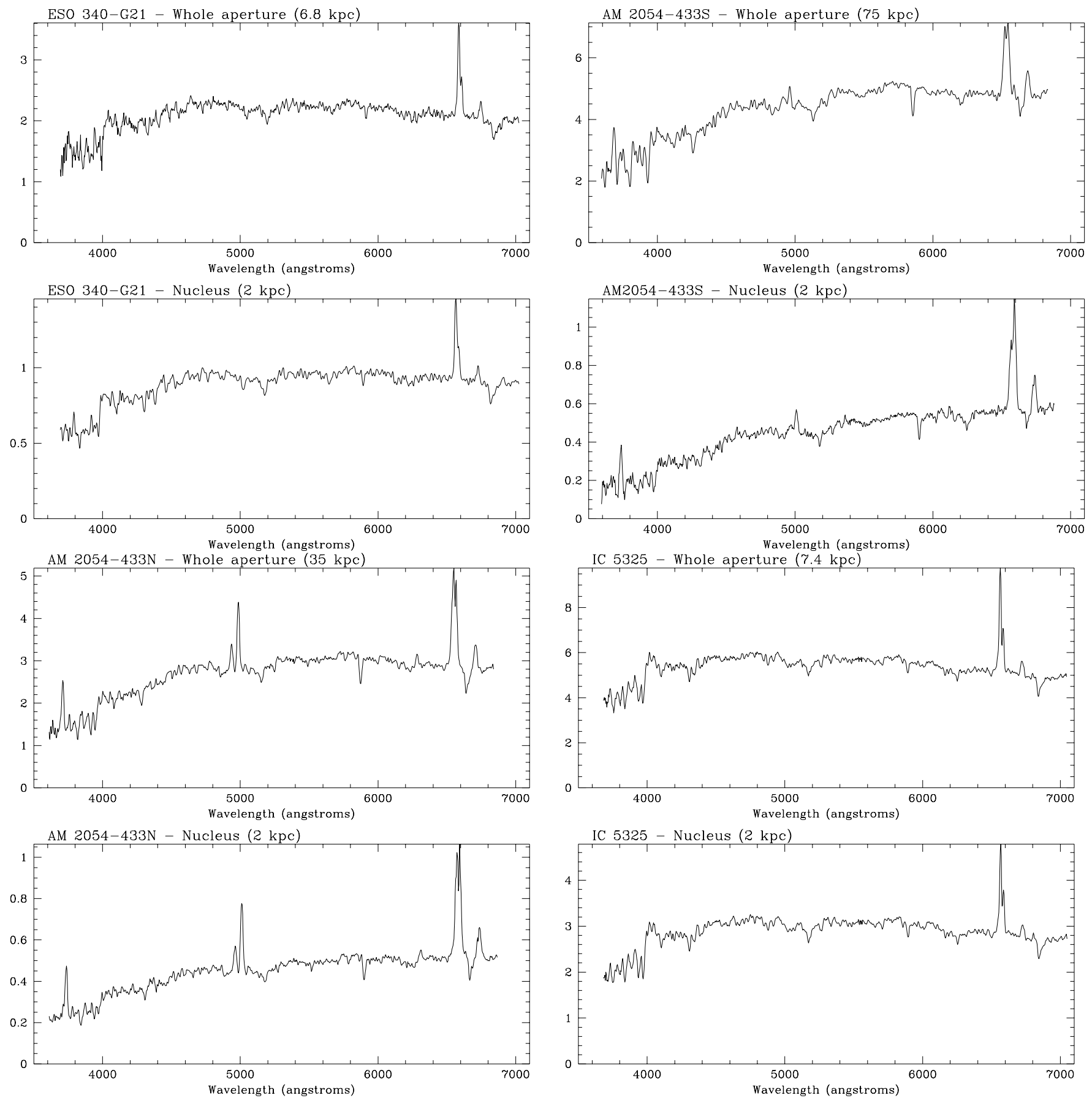

Fig. 4. continued

very prominent an it exhibits a shell-like structure in the $\mathrm{S}$ side.

The interesting $V-I$ color map of this galaxy can be appreciated in Fig. 5. It shows three well different regions with rather uniform colors: an inner region $\left(0^{\prime \prime}<r<15^{\prime \prime}\right)$ with $(V-I)=1.4$, an intermediate region $\left(15^{\prime \prime}<r<30^{\prime \prime}\right)$ with $(V-I)=1.2$ and the outer most region with the same color as the inner region. This remarkable structure could be signature of different events of stellar formation.

Unfortunately we could not obtain the $B-I$ and $B-V$ color maps due to poor seeing in the $B$ frame.

The luminosity profiles (Fig. 2f) show a weak bulge and a prominent disk with a feature located at $r=18^{\prime \prime}$ in the $B$ profile. In the $V, R$ and $I$ profiles this feature is present at $r=12^{\prime \prime}$. This excess is more prominent in the blue and it fades through redder bands.

Although this galaxy had been previously classified by $\mathrm{S} \& \mathrm{~W}$ as a LINER, we report a Seyfert 2 nucleus, as 
Table 7. Emission spectra

\begin{tabular}{lccccc}
\hline Galaxy & $\begin{array}{c}{[\mathrm{OII}]^{\mathrm{a}}} \\
\mathrm{EW}(\AA)\end{array}$ & $\begin{array}{c}\mathrm{H} \alpha+[\mathrm{NII}]^{\mathrm{a}} \\
\mathrm{EW}(\AA)\end{array}$ & $\begin{array}{r}{[\mathrm{OII}]^{\mathrm{b}}} \\
\mathrm{EW}(\AA)\end{array}$ & $\begin{array}{c}\mathrm{H} \alpha+[\mathrm{NII}]^{\mathrm{b}} \\
\mathrm{EW}(\AA)\end{array}$ & $\begin{array}{c}c z \\
\left(\mathrm{~km} \mathrm{~s}^{-1}\right)\end{array}$ \\
\hline ESO 316-G029 & 19 & 18 & 19 & 22 & $5654 \pm 70$ \\
NGC 3250E & 30 & 58 & 27 & 59 & $2737 \pm 40$ \\
ESO 317-G041 & 17 & 47 & 21 & 58 & $5708 \pm 60$ \\
NGC 3318 & 6 & 24 & 6 & 26 & $2706 \pm 60$ \\
NGC 3378 & 3 & 8 & - & 4 & $4858 \pm 70$ \\
ESO 266-G015 & 14 & 59 & 17 & 63 & $3122 \pm 40$ \\
ESO 340-G021 & - & 18 & 3 & 15 & $2683 \pm 50$ \\
AM 2054-433 N & 12 & 29 & 19 & 37 & $9706 \pm 50$ \\
AM 2054-433 S & 8 & 21 & 40 & 39 & $9297 \pm 50$ \\
IC 5325 & 2 & 20 & - & 17 & $1581 \pm 60$ \\
\hline
\end{tabular}

${ }^{\text {a }}$ whole aperture

b nuclear.

Table 8. Flux ratios

\begin{tabular}{lcccccc}
\hline Galaxy & $E(B-V)$ & {$[\mathrm{OII}] / \mathrm{H} \alpha$} & {$[\mathrm{OIII}] / \mathrm{H} \beta$} & {$[\mathrm{OI}] / \mathrm{H} \alpha$} & {$[\mathrm{NII}] / \mathrm{H} \alpha$} & {$[\mathrm{SII}] / \mathrm{H} \alpha$} \\
\hline ESO 316-G029 & 1.25 & 4.04 & 2.95 & 0.08 & 0.72 & 0.23 \\
NGC 3250E & 0.18 & 0.98 & 0.70 & 0.02 & 0.19 & 0.31 \\
ESO 317-G041 & 0.59 & 0.59 & 1.94 & 0.05 & 0.56 & 0.38 \\
ESO 266-G015 & 0.25 & 0.54 & 0.17 & 0.03 & 0.26 & 0.25 \\
AM 2054-433N & 0.11 & 1.38 & 2.44 & - & 3.53 & 1.45 \\
AM 2054-433S & 0.40 & 1.55 & 5.30 & 0.25 & 1.64 & 0.81 \\
\hline
\end{tabular}

inferred from the line flux ratios calculated from the pureemission spectrum (see Table 8).

We trace the rotation curve of this galaxy up to a radius of $20 \mathrm{Kpc}$, and it can be seen from Fig. 3d that the maximum velocity, corrected for the galaxy inclination $(b / a=0.5)$, is $v_{\max }=360 \pm 50 \mathrm{~km} \mathrm{~s}^{-1}$ at $r=5 \mathrm{kpc}$.

AM 2054-433S: We classify this object as a normal edge-on Sc galaxy as it can be judged from Fig. 1g. This galaxy shows a bright stellar-nucleus and two well developed arms. The NE arm is larger than the SW and it can be noticed several blue knots, $(B-V) \sim 0.5-0.6$, and dust lanes. It can also be seen from Fig. $1 \mathrm{~g}$ that this galaxy exhibits a disk warped towards the SO galaxy, probably due to the interaction.

The luminosity profiles are rather uniform in all bands (see Fig. 2g). Both the bulge and the disk of this galaxy are very prominent. Using the decomposition technique we obtain $r_{\mathrm{e}}=9.0 \mathrm{Kpc}$ and $D_{\mathrm{L}}=27.0 \mathrm{Kpc}$.

The flux line ratios calculated from the pure emission spectrum indicate a LINER-type nucleus, as previously found by S\&W.
From both $\mathrm{H} \alpha$ and [NII] emission lines we provide the rotation curve of this galaxy up to a radius of $40 \mathrm{kpc}$ which is shown in Fig. 3e. The rotation curve corrected for the galaxy inclination shows a maximum $v_{\max }=375 \pm$ $50 \mathrm{~km} \mathrm{~s}^{-1}$ at $r=10 \mathrm{kpc}$. This high rotational velocity is similar to that corresponding to AM 2054-433 N.

IC 5325: From the $B$ image shown in Fig. 1 h we conclude that this is a normal face-on Sb galaxy. The galaxy shows a stellar-nucleus from which several arms emerge. The arms are rather fragmented and they exhibit several blue knots with $(B-V)=0.2-0.6$. The galaxy colors are rather uniform and the luminosity profiles are clearly disk dominated beyond $r=5^{\prime \prime}$ (see Fig. 2h).

The integrated spectrum of the galaxy shown in Fig. 4j corresponds to a Sb type and we obtain $\mathrm{EW}(\mathrm{H} \alpha+$ $[\mathrm{NII}])=20 \AA$, a typical value for this type of galaxies. 


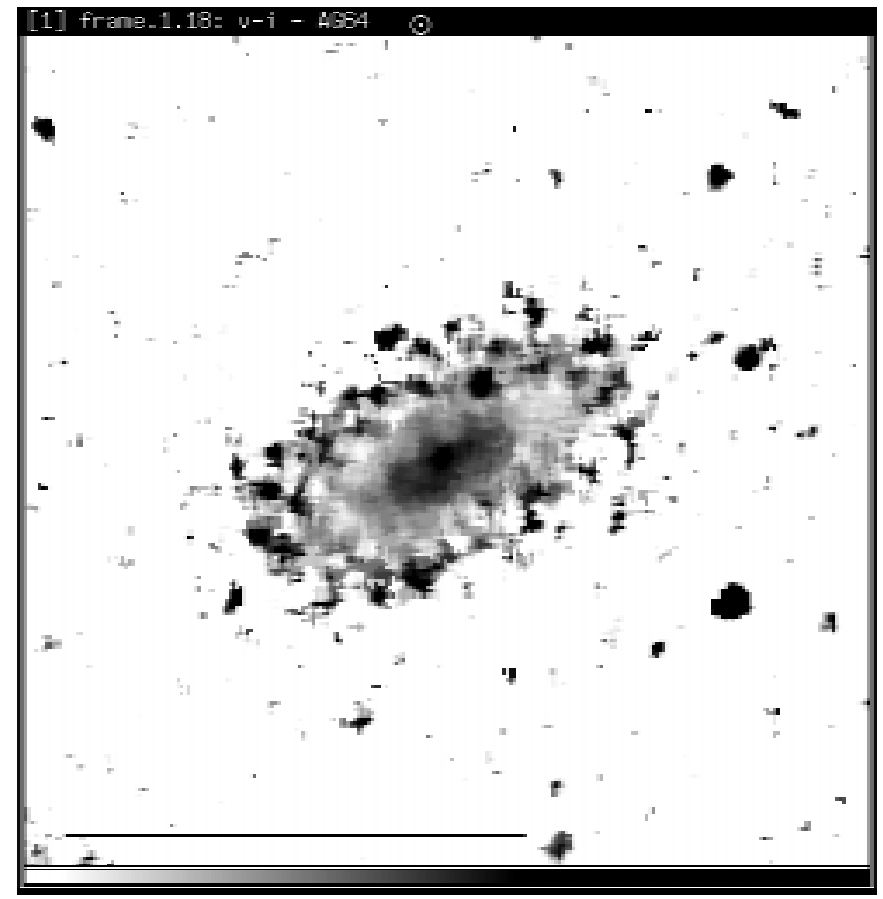

Fig. 5. $V-I$ color map of the galaxy AM 2054-433N. North is at top and East to the left. The lower left bar indicates 1 arcmin

\section{Concluding remarks}

Throughout this paper we have discussed the photometric and spectroscopic properties of a sample of galaxies selected from the Agüero Catalogue of Peculiar Galaxies.

From the analysis of the images we conclude that only 4 of the selected objects are truly peculiar galaxies, namely: NGC 3250E, ESO 317-G41, NGC 3318 and ESO 266-G13. The rest of the sample consists in 5 normal spirals, ESO 316-G29, NGC 3378, ESO 340-G21, AM 2054-433 S, ESO 286-G17, and one SO galaxy, AM 2054-433 N.

A more detailed analysis of the images does not reveal signs of mergers in the peculiar galaxies. Moreover, we could not find companions for these galaxies in a $10^{\prime} \times 10^{\prime}$ field.

The luminosity profiles of normal and peculiar galaxies follow the $r^{1 / 4}+$ exponential law except NGC 3250E. This galaxy shows a peculiar luminosity profile which clearly exhibits two exponential components, probably due to the presence of two different discs.

It is also observed that the luminosity profiles of normal and peculiar galaxies show features that are signatures of subsystems and star forming regions distributed throughout the galaxies. In this sense, it is interesting to observe that peculiar galaxies are indistinguishable, at least from a purely photometric point of view, from the normal ones. Most of the galaxies of the sample show from the $B-V$ map several blue regions with $(B-V)$ colors ranging from 0.2 to 0.6 indicating ongoing star formation. An interesting case is AM 2054-433 N which exhibits a $V-I$ map that suggests star formation events in three different annulus concentric to the nucleus.

From the spectroscopic analysis we report a LINERtype nucleus for the normal spiral ESO 316-G29 and a Seyfert 2 galaxy, AM 2054-433 N. We also confirm one LINER-type spectrum for the spiral component of the interacting pair AM 2054-433. This fact was possible since we could subtract the underlying stellar signatures using a database of early type galaxy spectra. This technique proved to be a powerful tool in order to search active galactic nuclei.

It is very suggesting that 3 of the 4 peculiar galaxies show a starburst type spectrum. Moreover, the EW $(\mathrm{H} \alpha+[\mathrm{NII}])$ values measured in the integrated spectra of the starburst galaxies indicate that these objects are under a global burst of star formation which occurred 10 Myr ago. For these galaxies we also find similar values EW $(\mathrm{H} \alpha+[\mathrm{NII}]) \sim 60 \AA$, which is three times higher than the observed average for the normal galaxies of the sample, $\sim 22 \AA$. We note that these starburst galaxies have EW $(\mathrm{H} \alpha+[\mathrm{NII}])$ comparable to those in samples of merging and interacting galaxies (L\&K, Donzelli \& Pastoriza 1997).

Although the origin of the mechanism that triggers star formation remains unclear, the analysis of these data suggests that the peculiar morphologies of these galaxies are closely related to global starbursts. However, due to the small size of our sample this idea is not conclusive. Further observations of peculiar galaxies are needed to support this hypothesis.

Acknowledgements. We are in debt with the staffs of CASLEO and UTSO for their excellent support. We acknowledge the very useful comments by the referee that greatly improved this paper. Many thanks to Dr. Diego García Lambas for the English revision. The authors acknowledge use of the CCD and data acquisition, system supported under U.S. National Science Foundation grant AST-90-15827, to R.M. Rich. This research has made use of the NASA/IPAC Extragalactic Database (NED) which is operated by the Jet Propulsion Laboratory, CALTECH, under contract with the National Aeronautics and Space Administration.

\section{References}

Agüero E.L., 1971, PASP 83, 310

Appleton P.N., Struck-Marcell C., 1987, ApJ 312, 566

Bica E., 1988, A\&A 195, 76

de Vaucouleurs G., de Vaucouleurs A., Corwin H.G. Jr., et al., 1991, Third Reference Catalogue of Bright Galaxies. Springer Verlag

Copetti M.V.F., Pastoriza M.G., Dottori H.A., 1986, A\&A 156, 111

Donzelli C.J., Pastoriza M.G., 1997, ApJS 111, 181

Donzelli C.J., Espíndola M., 1996, AJ 111, 1057

Dressler A., Oemler Jr. A., Butcher H.R., Gunn J.E., 1994, ApJ 430, 107 
Gallagher J.S., Hunter D.A., 1987, AJ 94, 43

Jedrzejewski R.I., 1987, MNRAS 226, 747

Kenney J.D.P., Rubin V., Planesas P., Young J.S., 1995, ApJ 438, 135

Kenney J.D.P., Koopmann R.A., 1996, AJ 111, 152

Kennicutt R.C., 1990, Paired and Interacting Galaxies, IAU

Colloquium, Sulenctic J.W. and Keel W.C. (eds.), No. 124, p. 269

Kennicutt R.C., 1992, ApJS 79, 255

Landolt A.U., 1992, AJ 104, 372

Leitherer C., Heckman T.M., 1995, ApJS 96, 9

Liu C.T., Kennicutt R.C., 1995, ApJ 450, 547

Marston A.P., 1988, MNRAS 230, 97

McCall M.L., Rybski P.M., Shields G.A., 1985, ApJS 57, 1

Moss C., Whittle M., 1993, ApJ 407, L17
Oemler A. Jr., 1992, in Clusters and Supercluster of Galaxies, Fabian A.C. (ed.). Kluwer, Dordrecht, p. 29

Osterbrock D.E., 1989, Astrophysics of Gaseous Nebulae and Active Galactic Nuclei. University Science Books, Mill Valley, CA

Persic M., Salucci P., 1995, ApJS 99, 510

Schweizer F., 1992, in Physics of Nearby Galaxies: Nature or Nurture, Thuan T.X., Balkowski C., and Thanh Van Tran (eds.). Editions Frontieres, Gif-Sur-Yvette Cedex, p. 283

Sekiguchi K., Wolstencroft R.D., 1992, MNRAS 255, 581

Shombert J.M., Bothun G.D., 1987, ApJ 92, 60

Stone R.P.S., Baldwin J.A., 1982, MNRAS 204, 347

Tonry J.L., Davies M., 1979, AJ 84, 1511

Veilleux S., Osterbrock D.E., 1987, ApJS 63, 295 